\title{
Dosimetric characterization of hypofractionated Gamma Knife radiosurgery of large or complex brain tumors versus linear accelerator-based treatments
}

\author{
Peng Dong, $\mathrm{PhD},{ }^{1,3}$ Angélica Pérez-Andújar, $\mathrm{PhD},{ }^{1}$ Dilini Pinnaduwage, $\mathrm{PhD},{ }^{4}$ \\ Steve Braunstein, MD, PhD, ${ }^{1}$ Philip Theodosopoulos, MD, ${ }^{2}$ Michael McDermott, MD, ${ }^{2}$ \\ Penny Sneed, MD, ${ }^{1}$ and Lijun Ma, PhD' ${ }^{1}$
}

Departments of ${ }^{1}$ Radiation Oncology and ${ }^{2}$ Neurosurgery, University of California, San Francisco; ${ }^{3}$ Department of Radiation Oncology, Stanford University, Stanford, California; and ${ }^{2}$ Department of Radiation Oncology, The University of Arizona School of Medicine and St. Joseph's Hospital and Medical Center, Phoenix, Arizona

OBJECTIVE Noninvasive Gamma Knife (GK) platforms, such as the relocatable frame and on-board imaging, have enabled hypofractionated GK radiosurgery of large or complex brain lesions. This study aimed to characterize the dosimetric quality of such treatments against linear accelerator-based delivery systems that include the CyberKnife (CK) and volumetric modulated arc therapy (VMAT).

METHODS Ten patients treated with VMAT at the authors' institution for large brain tumors $>3 \mathrm{~cm}$ in maximum diameter) were selected for the study. The median prescription dose was $25 \mathrm{~Gy}$ (range 20-30 Gy) in 5 fractions. The median planning target volume (PTV) was $9.57 \mathrm{~cm}^{3}$ (range 1.94-24.81 $\left.\mathrm{cm}^{3}\right)$. Treatment planning was performed using Eclipse External Beam Planning V11 for VMAT on the Varian TrueBeam system, Multiplan V4.5 for the CyberKnife VSI System, and Leksell GammaPlan V10.2 for the Gamma Knife Perfexion system. The percentage of the PTV receiving at least the prescription dose was normalized to be identical across all platforms for individual cases. The prescription isodose value for the PTV, conformity index, Paddick gradient index, mean and maximum doses for organs at risk, and normal brain dose at variable isodose volumes ranging from the 5-Gy isodose volume (V5) to the 15-Gy isodose volume (V15) were compared for all of the cases.

RESULTS The mean Paddick gradient index was $2.6 \pm 0.2,3.2 \pm 0.5$, and $4.3 \pm 1.0$ for GK, CK, and VMAT, respectively $(p<0.002)$. The mean V15 was $7.5 \pm 3.7 \mathrm{~cm}^{3}$ (range 1.53-13.29 $\left.\mathrm{cm}^{3}\right), 9.8 \pm 5.5 \mathrm{~cm}^{3}$ (range 2.07-18.45 $\left.\mathrm{cm}^{3}\right)$, and $16.1 \pm 10.6 \mathrm{~cm}^{3}$ (range 3.58-36.53 $\mathrm{cm}^{3}$ ) for GK, CK, and VMAT, respectively ( $\mathrm{p} \leq 0.03$, paired 2-tailed t-tests). However, the average conformity index was $1.18,1.12$, and 1.21 for GK, CK, and VMAT, respectively $(p>0.06)$. The average prescription isodose values were $52 \%$ (range $47 \%-69 \%$ ), 60\% (range 46\%-68\%), and $88 \%$ (range $70 \%-94 \%$ ) for GK, $\mathrm{CK}$, and VMAT, respectively, thus producing significant variations in dose hot spots among the 3 platforms. Furthermore, the mean V5 values for GK and CK were similar $(p>0.79)$ at $71.9 \pm 36.2 \mathrm{~cm}^{3}$ and $73.3 \pm 31.8 \mathrm{~cm}^{3}$, respectively, both of which were statistically lower $(p<0.01)$ than the mean V5 value of $124.6 \pm 67.1 \mathrm{~cm}^{3}$ for VMAT.

CONCLUSIONS Significantly better near-target normal brain sparing was noted for hypofractionated GK radiosurgery versus linear accelerator-based treatments. Such a result supports the use of a large number of isocenters or confocal beams for the benefit of normal tissue sparing in hypofractionated brain radiosurgery.

http://thejns.org/doi/abs/10.3171/2016.7.GKS16881

KEY WORDS hypofractionation; stereotactic radiosurgery; volumetric modulated arc therapy; Gamma Knife; CyberKnife; normal tissue; oncology

\footnotetext{
ABBREVIATIONS $\mathrm{Cl}=$ conformity index; $\mathrm{CK}=$ CyberKnife; f-IMRT = fan beam-based intensity modulated radiotherapy; $\mathrm{GI}=$ gradient index; $\mathrm{GK}=\mathrm{Gamma}$ Knife; $\mathrm{LINAC}$ $=$ linear accelerator; PTV = planning target volume; VMAT = volumetric modulated arc therapy; V5 = 5-Gy isodose volume; V10 = 10-Gy isodose volume; V15 = 15-Gy isodose volume. 
$\mathrm{G}$ AMMA Knife (GK) radiosurgery has been the gold standard therapy for single-fraction high-dose irradiation of relatively small brain lesions (e.g., approximately $<4 \mathrm{~cm}$ in maximum diameter). ${ }^{1,24,25}$ However, recent technical advancements in noninvasive platforms, such as the relocatable eXtend head frame system ${ }^{13,26}$ and on-board $\mathrm{kV}$ imaging capabilities, ${ }^{22}$ have enabled the GK to be used to perform hypofractionated radiosurgery of large and/or complex intracranial lesions that had been mostly treated with linear accelerator (LINAC)-based modalities, such as X-band CyberKnife (CK) or S-band LINACs.

Compared with LINAC-based modalities, major physical characteristics that distinguish GK include the following: 1) GK uses a narrow energy spectrum of gamma rays from Co-60 sources instead of broad-spectrum bremsstrahlung of electrons hitting high- $Z$ targets from a LINAC; and 2) treatment of a large target via GK requires many isocenters, collectively involving thousands of beams from different directions, whereas CK typically uses nonisocentric beams of approximately 100-300 directions and traditional S-band LINACs tend to use a single isocenter with approximately 5-10 fixed beam directions and/or 1-10 rotational arc beams.

Previous investigations that compared GK treatment planning quality against LINAC-based modalities for treating sizable lesions were largely constrained by the practical consideration of the invasive metal frame required for GK treatments. However, 1 study, which characterized GK treatments of intermediate-size $(2-4 \mathrm{~cm})$ intracranial targets versus fan beam-based intensity modulated radiotherapy (f-IMRT) treatments, found that GK spares the normal brain tissue significantly better, spurring the concern for pediatric patients. ${ }^{16}$

With rapid advancements in digitally controlled LINAC delivery, such as volumetric modulated arc therapy (VMAT) via flattening filter-free beams, ${ }^{18}$ studies have reported feasibilities of applying such treatments toward

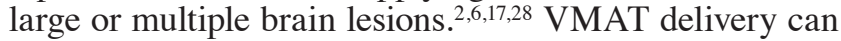
be, in theory, viewed as intensity modulated arc therapy delivery with multiple overlapping arcs in analogous f-IMRT delivery. ${ }^{31,32}$ Therefore, the question arises as to whether VMAT delivery has overcome the normal tissue-sparing discrepancy compared with GK delivery, as found with f-IMRT delivery. This question is especially relevant for an on-board image-guided GK system for which hypofractionated image-guided treatments of large brain targets have become a turn-key solution in contrast to the previous GK systems., ${ }^{9,21}$ As a result, the goal of our study was to investigate dosimetric capabilities of hypofractionated GK treatments of larger or complex brain tumors versus the latest LINAC-based treatments with either CK or S-band LINAC-based VMAT treatment deliveries.

\section{Methods}

\section{Patient Selection}

Ten patients with single cranial tumors, who were originally treated at our institution with the VMAT technique (RapidArc, Varian Oncology Systems), were selected for this study (Table 1). These VMAT cases were patients who were consecutively treated between 2014 and 2015. The median prescription dose was 25 Gy (range 20-30 Gy) in 5 fractions. The median planning target volume (PTV) was $9.57 \mathrm{~cm}^{3}$ (range 1.94-24.81 $\mathrm{cm}^{3}$ ). The median dose coverage was $97 \%$ (range 92\%-99\%) for all of the cases. Patients who underwent hypofractionated treatments were largely randomly selected in terms of patient performance status, primary versus metastatic disease, target location, and nearby critical structures.

For each case, the DICOM-radiotherapy (DICOM-RT) structure sets were extracted and exported to both the GK and CK treatment planning systems (described below) by experienced users in a blinded and independent fashion, i.e., the participants planning 1 modality (e.g., GK, CK, or VMAT) were blinded to the treatment planning process and quality of the other 2 modalities when planning each case. Once completed, all of the cases were collected on a centralized system (MIM Software, Version 6) for final review and analysis.

\section{Treatment Planning and Delivery System}

The VMAT treatment planning was performed for all of the cases via the Eclipse External Beam Planning V11 for the TrueBeam STx LINAC system (Varian Oncology Systems) equipped with a high-definition 120-leaf MLC system. The center leaf size was $2.5 \mathrm{~mm}$ and peripheral leaves were $5 \mathrm{~mm}$. Following the general recipe of VMAT delivery, 2 arcs of isocentric full $360^{\circ}$ coplanar beams with alternate collimator angles of $30^{\circ}$ or $330^{\circ}$ were used, with sections encompassing critical organs blocked when planning a treatment. The isocenter of the plan was all placed inside the individual solitary target.

Note that the use of coplanar arc beams is not mandatory for cranial VMAT treatments. However, they are adopted for patient treatments at our institution due to marginal differences observed between the coplanar technique and the noncoplanar techniques for single-target treatments, which was noted in our previous study. ${ }^{6}$ Furthermore, Eclipse uses the progressive resolution optimizer with explicit dose-volume histogram objectives. To decrease dose spillage, the planner iteratively tunes the weighting on the normal tissue objective, which controls how dose falls off outside a defined PTV. The optimization typically took 25-35 minutes per arc. The delivery of the plan is approximately 5 minutes for 6-MV standard flattened beams or approximately half of that time for 6-MV flattening filterfree beams. ${ }^{6,30}$

The CK treatment planning was performed via the Multiplan V4.5 for the CyberKnife VSI System via sequential inverse optimization. The system is equipped with both fixed-size cone and iris collimators with apertures varying from 5 to $60 \mathrm{~mm}$ and is capable of nonisocentric, noncoplanar delivery. Two fixed-size cone collimators were used for each plan to reduce delivered monitor units compared with using 1 collimator. Collimators were chosen such that 1 collimator diameter was approximately equal to the central part of the lesion and the other was small enough to cover the tumor's smallest features.

For treatment node selection, we used the default template path set for the head location: "1path_head." The CK 
TABLE 1. Physical and dosimetric characteristics of the 10 cases selected for the study

\begin{tabular}{rccccrc}
\hline $\begin{array}{c}\text { Case } \\
\text { No. }\end{array}$ & $\begin{array}{c}\text { Dose, } \\
\text { Gy }\end{array}$ & $\begin{array}{c}\text { No. } \\
\text { of Fx }\end{array}$ & $\begin{array}{c}\text { Target } \\
\text { Vol, cm }\end{array}$ & $\begin{array}{c}\text { Target } \\
\text { Coverage, } \%^{*}\end{array}$ & $\begin{array}{r}\text { PIV, } \\
\mathrm{cm}^{3}\end{array}$ & $\begin{array}{c}\mathrm{PIV}_{50}, \\
\mathrm{~cm}^{3}\end{array}$ \\
\hline 1 & 30 & 5 & 8.0 & 98 & 8.8 & 41.8 \\
\hline 2 & 25 & 5 & 13.0 & 97 & 13.4 & 57.3 \\
\hline 3 & 25 & 5 & 10.1 & 95 & 10.4 & 42.6 \\
\hline 4 & 25 & 5 & 8.6 & 95 & 9.3 & 44.6 \\
\hline 5 & 20 & 5 & 6.4 & 95 & 6.6 & 19.0 \\
\hline 6 & 20 & 5 & 24.8 & 99 & 30.3 & 88.1 \\
\hline 7 & 25 & 5 & 11.7 & 98 & 12.7 & 55.9 \\
\hline 8 & 25 & 5 & 9.1 & 98 & 14.7 & 89.3 \\
\hline 9 & 25 & 5 & 15.3 & 92 & 16.7 & 90.3 \\
\hline 10 & 25 & 5 & 1.9 & 99 & 3.5 & 12.5 \\
\hline
\end{tabular}

$\mathrm{Fx}=$ total number of fractions; $\mathrm{PIV}=$ prescription isodose volume, i.e., total isodose volume enclosed by the prescription isodose surface for the reference VMAT treatment plans; PIV $_{50}=$ total isodose volume enclosed by $50 \%$ of the prescription isodose surface for the reference VMAT treatment plans.

* The target coverage was normalized to be identical across all of the treatment modalities.

system allows for 12 beam angles at each node position. Approximately 50-100 nodes and 200-250 beams were used in our plans. Several "auto-shells" were created outside the target volume to constrain the conformity and the extent of the low-dose region. Beam-reduction and timereduction steps were used to confine the treatment time to $<40$ minutes. The treatment planning optimization time was approximately $<1$ hour.

The GK treatment planning was performed via the Leksell GammaPlan V10.2 for the Perfexion eXtend system. ${ }^{3,13,26}$ Dose distribution of each shot is shaped from 192 directions through 8 sectors of independently opened or blocked collimators in the size of 4,8 , and $16 \mathrm{~mm}$. Manual forward planning with the GammaPlan was performed. Treatment planning employs a compromise between tight dose gradients and treatment time because the benefit of tight dose gradients achieved by using smaller collimator sizes is offset by the long treatment time (approximately 20-30 minutes). The optimization time depends on the planner's skill and experience with the system and typically took 10-20 minutes.

\section{Plan Evaluation}

The percentage of the PTV receiving at least the prescription dose ranged from $95 \%$ to $99 \%$ and was normalized to be identical across all of the platforms for individual cases (Table 1). The prescription isodose values were between $70 \%$ and $94 \%$ for VMAT, $47 \%$ and $69 \%$ for GK, and $46 \%$ and $68 \%$ for CK. Note that the prescription isodose values for the CK and GK treatments were selected for the purpose of matching the PTV coverage of the original VMAT treatment plans that were delivered for patient treatments. Special care was taken to ensure that any dose larger than $105 \%$ of the prescription dose was located within the PTV. The VMAT and CK systems consider low-dose spillage as a significant penalty in the optimization process, whereas for GK all of the shots are placed manually. The dose limits to critical structures (brain, eyes, optic nerves, chiasm, brainstem, spinal cord, cochlea, and pituitary gland) were also monitored for the 3 modalities during plan generation.

The following dosimetry metrics were analyzed: 1) conformity index (CI), which is the ratio of the volume covered by the prescription isodose (V100\%) to the PTV volume (VPTV) as normalized by the target volume coverage (the value is typically between 1 and 1.5 and is equal to the inverse of the Paddick $\mathrm{CI}^{19}$ ); 2) Paddick gradient index (GI), which is defined as the ratio of the $50 \%$ prescription dose isodose volume to the $100 \%$ prescription dose isodose volume; ${ }^{20}$ and 3) dose-volume parameters, with normal brain dose at variable isodose volumes, such as 5-Gy isodose volume (V5), 10-Gy isodose volume (V10), and 15-Gy isodose volume (V15). The normal brain volume is defined as the entire brain volume excluding the PTV. Finally, paired nonparametric ANOVA tests were performed to examine the statistical significance of the differences seen across these modalities for the above dosimetric indices.

\section{Results}

Figure 1 shows the isodose distribution for a representative case from the 3 modalities in the axial, sagittal, and coronal planes. The differences in the plans from the 3 modalities are qualitatively demonstrated in this representative case. With equivalent coverage between all techniques required at planning, the larger low-dose $(<5 \mathrm{~Gy})$ volume in the VMAT cases and smaller medium-dose (approximately 10-Gy level) volume for GK and CK plans are evident in this example representation.

Figure 2A shows the distribution for the CI. The average CI was 1.18, 1.12, and 1.21 for GK, CK, and VMAT, respectively $(p>0.06)$. Figure $2 B$ shows the distribution for the GI. The mean Paddick GI was $2.6 \pm 0.2,3.2 \pm 0.5$, and $4.3 \pm 1.0$ for the GK, CK, and VMAT, respectively ( $\mathrm{p}<$ $0.002)$. Figure $2 \mathrm{C}$ details the distribution for the prescription isodose line. The average prescription isodose values were $52 \%$ (range $47 \%-69 \%$ ), 60\% (range $46 \%-68 \%$ ), and $88 \%$ (range 70\%-94\%) for GK, CK, and VMAT, respectively, thus producing significant variations in the dose hot spots among the 3 platforms.

Figure 3 represents distributions for the normal brain volumes receiving a dose of $15 \mathrm{~Gy}, 10 \mathrm{~Gy}$, and $5 \mathrm{~Gy}$. The mean V15 was $7.5 \pm 3.7 \mathrm{~cm}^{3}$ (range $1.53-13.29 \mathrm{~cm}^{3}$ ), 9.8 $\pm 5.5 \mathrm{~cm}^{3}$ (range 2.07-18.45 $\mathrm{cm}^{3}$ ), and $16.1 \pm 10.6 \mathrm{~cm}^{3}$ (range $3.58-36.53 \mathrm{~cm}^{3}$ ) for GK, CK, and VMAT, respectively ( $\mathrm{p} \leq 0.03)$. The mean V10 values for GK and CK were similar $(\mathrm{p}>0.13)$ at $18.1 \pm 8.8 \mathrm{~cm}^{3}$ and $20.5 \pm 10.1$ $\mathrm{cm}^{3}$, respectively, both of which were statistically lower $(\mathrm{p}<0.01)$ than the mean V10 value of $37.8 \pm 21.0 \mathrm{~cm}^{3}$ for VMAT. Furthermore, the mean V5 values for GK and CK were similar $(\mathrm{p}>0.79)$ at $71.9 \pm 36.2 \mathrm{~cm}^{3}$ and $73.3 \pm 31.8$ $\mathrm{cm}^{3}$, respectively, both of which were statistically lower $(\mathrm{p}<0.01)$ than the mean V5 value of $124.6 \pm 67.1 \mathrm{~cm}^{3}$ for VMAT. Note that the whisker-box-plotted range of Figs. 2 and 3 exhibited greater variations for VMAT compared with GK or CK. Such variations were not found to cor- 


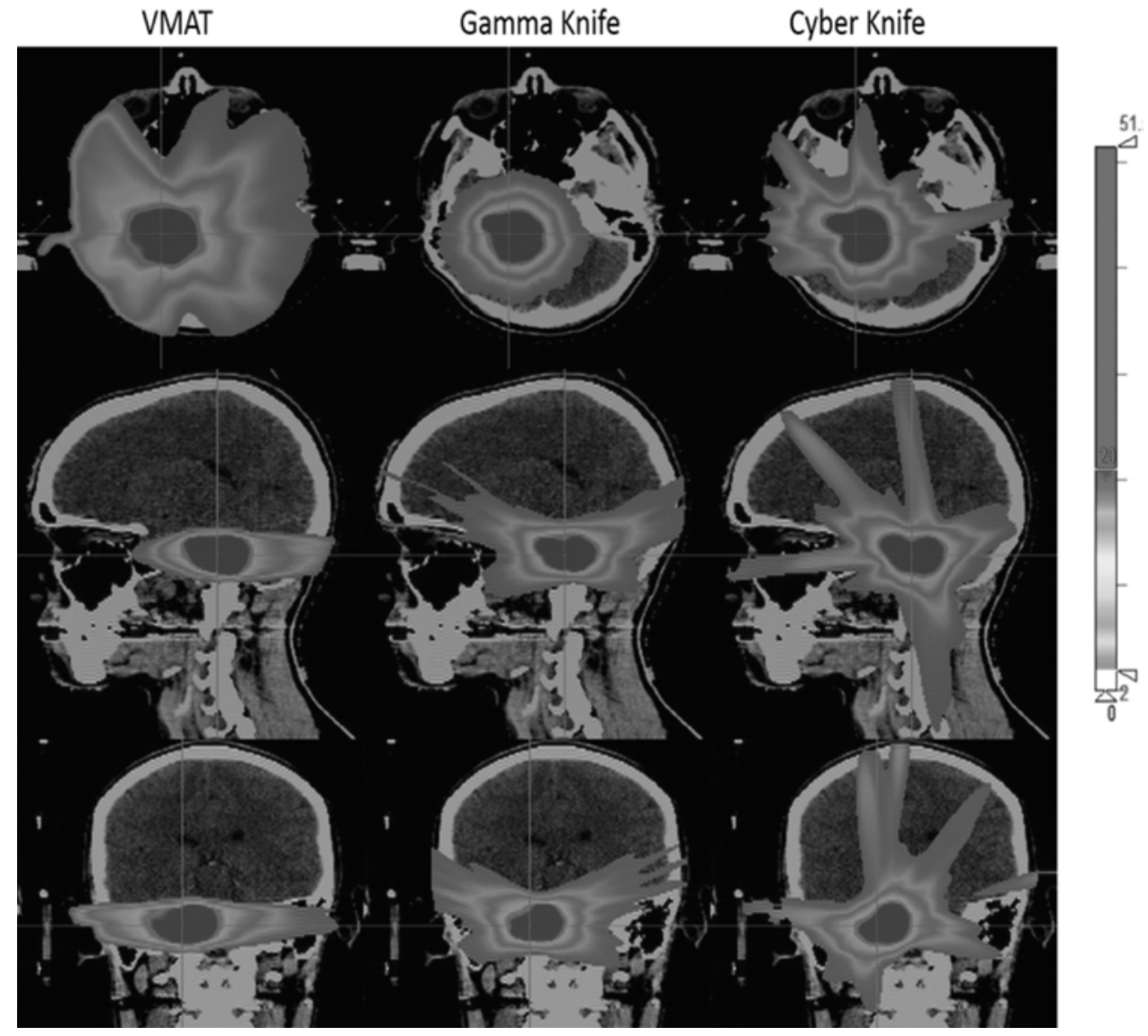

FIG. 1. Dose distributions of VMAT, GK, and CK plans in the axial plane (upper), sagittal plane (center), and coronal plane (lower) for a typical patient.

relate with the size, geometry, or location of the targets, and the effect may be contributed by the small sample size of the study.

Finally, the doses to the critical structures were all found to be within the clinical constraints and were nonremarkable for the 3 modalities ( $p>0.05$ ). Certain variations noted for small structures (such as the lens of the eye) were probably a contribution from beamlets passing through the structure, because they were not specifically constrained during planning for the current study. The beam-on time was estimated to be approximately 40 minutes, 30 minutes, and 5 minutes under a nominal dose rate of $3.0 \mathrm{~Gy} / \mathrm{min}, 10.0 \mathrm{~Gy} / \mathrm{min}$, and $20.0 \mathrm{~Gy} / \mathrm{min}$ for GK, $\mathrm{CK}$, and VMAT, respectively, excluding patient setup and treatment-related quality assurance time and effort.

\section{Discussion}

Large statistically significant differences in dose falloff (e.g., as indicated by the GI values for equivalent target volume coverage and dose conformity) have been observed for hypofractionated radiotherapy of large brain tumors among GK, CK, and VMAT treatments. Compared with LINAC-based CK/VMAT deliveries, GK consistently produced sharper dose fall-off and better normal brain-sparing results, despite greater central tar- get dose. Such results were in good agreement with studies that treated multiple lesions with these platforms ${ }^{11,12}$ and an early study ${ }^{16}$ that compared GK against f-IMRT deliveries for treatment of intermediate-sized targets, where GK was found to produce significantly sharper dose fall-off in sparing the normal brain tissue surrounding the target.

In the context of hypofractionated treatments for large brain tumors, our study has suggested that there is ample room for future technical improvements in LINAC-based as well as GK-based hypofractionated treatments. Techniques such as the effective utilization of noncoplanar arc beams via manual or broad-range optimization approaches, ${ }^{4,6,29}$ 2-step optimizations for CK beams,${ }^{14}$ and optimizing sector beam-based intensity modulated GK beams ${ }^{10}$ have all shown promise for improving the existing treatment-planning qualities of VMAT, CK, and GK, respectively. The results of the present study, which are based on default and repeatable techniques, have established a reference baseline for evaluating the aforementioned and other potential future developments.

The results of our study also point out the general fallacy of assuming that the same dose to the target periphery would produce an equivalent normal brain tissue dose from one treatment platform to another. This issue is particularly relevant for large lesion treatments because the 

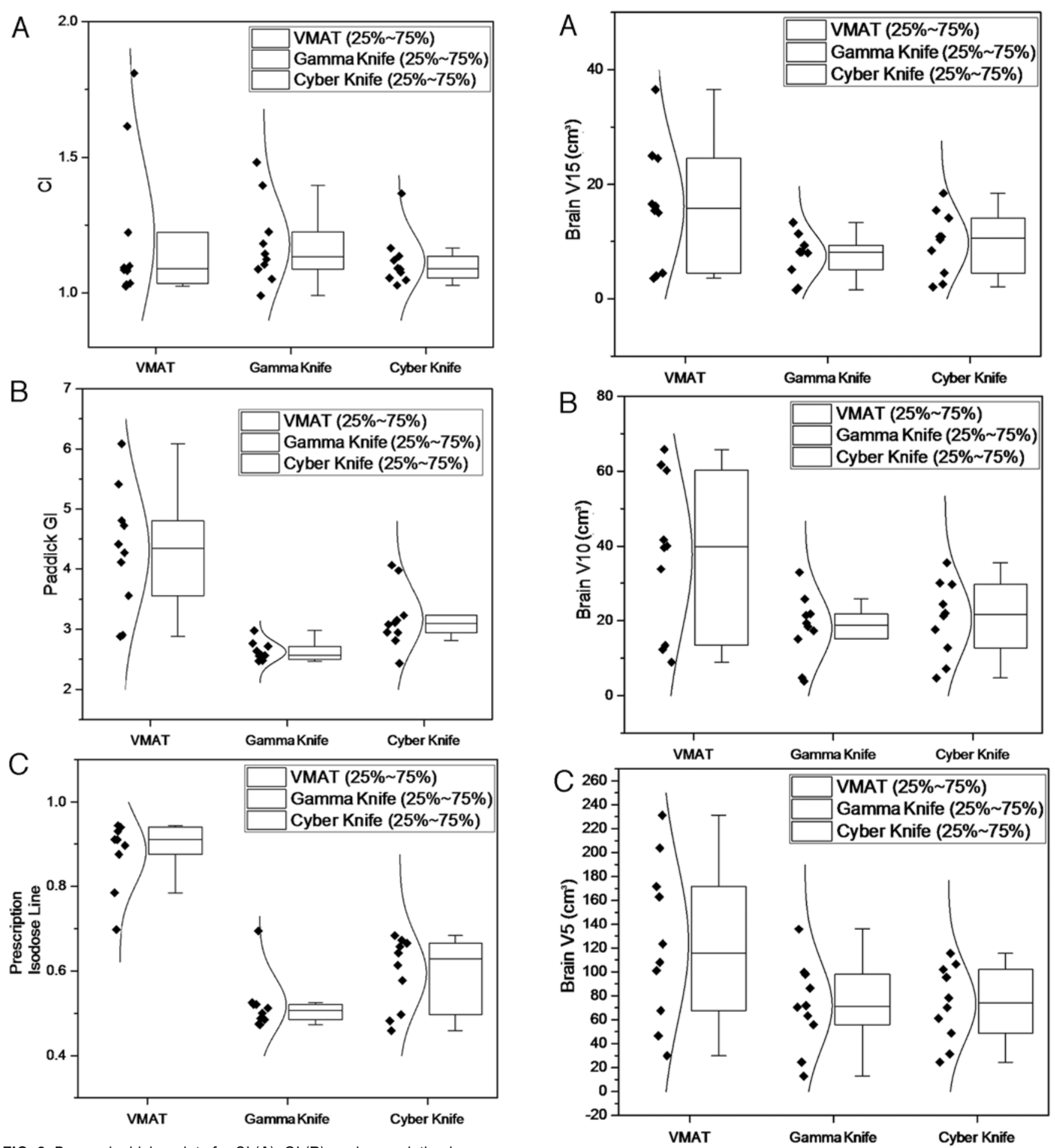

FIG. 2. Box-and-whisker plots for $\mathrm{Cl}(\mathrm{A}), \mathrm{GI}(\mathrm{B})$, and prescription isodose (C) values for all of the cases planned for the GK, CK, and VMAT treatments.

dose to the normal brain has been found to increase steeply in a nonlinear function as the target size increases. ${ }^{15}$ Ongoing and further clinical studies investigating the normal brain-tolerable dose for hypofractionated treatments (such as addressing the questions like what the

10-Gy or 12-Gy single-fractional equivalent doses are for different hypofractional schema, and whether biological equivalent dose-based conversion formula of our previous study ${ }^{5}$ equating hypofractionated treatments such as 25 Gy in 5 fractions with single-fractional equivalent dose 
remains valid, and so on) will ultimately help to elucidate the best dosing and fractionation practices, not only based on the dose prescribed to the target periphery but also taking into account the dose to the surrounding normal brain tissues. ${ }^{8,23,27}$ Until such studies are completed, we caution and recommend clinical users to pay special attention to the normal brain dose and margin status in the vicinity of a large or complex brain target receiving hypofractionated radiosurgery.

\section{Conclusions}

When treating large or complex brain lesions via hypofractionated radiosurgery, GK better spares the normal brain and delivers higher target dose compared with LINAC-based CK/VMAT deliveries.

\section{References}

1. Andrews DW, Bednarz G, Evans JJ, Downes B: A review of 3 current radiosurgery systems. Surg Neurol 66:559-564, 2006

2. Clark GM, Popple RA, Prendergast BM, Spencer SA, Thomas EM, Stewart JG, et al: Plan quality and treatment planning technique for single isocenter cranial radiosurgery with volumetric modulated arc therapy. Pract Radiat Oncol 2:306-313, 2012

3. Devriendt D, De Smedt F, Glineur R, Massager N: Fivefraction Gamma Knife radiosurgery using the Extend relocatable system for benign neoplasms close to optic pathways. Pract Radiat Oncol 5:e119-e125, 2015

4. Dong P, Lee P, Ruan D, Long T, Romeijn E, Low DA, et al: $4 \pi$ noncoplanar stereotactic body radiation therapy for centrally located or larger lung tumors. Int J Radiat Oncol Biol Phys 86:407-413, 2013

5. Fogh S, Ma L, Gupta N, Sahgal A, Nakamura JL, Barani I, et al: High-precision volume-staged Gamma Knife surgery and equivalent hypofractionation dose schedules for treating large arteriovenous malformations. J Neurosurg 117 Suppl:115-119, 2012

6. Hossain S, Keeling V, Hildebrand K, Ahmad S, Larson DA, Sahgal A, et al: Normal brain sparing with increasing number of beams and isocenters in volumetric-modulated arc beam radiosurgery of multiple brain metastases. Tech Cancer Res Treat [epub ahead of print], 2015

7. Kilby W, Dooley JR, Kuduvalli G, Sayeh S, Maurer CR Jr: The CyberKnife robotic radiosurgery system in 2010. Technol Cancer Res Treat 9:433-452, 2010

8. Lawrence YR, Li XA, el Naqa I, Hahn CA, Marks LB, Merchant TE, et al: Radiation dose-volume effects in the brain. Int J Radiat Oncol Biol Phys 76 (3 Suppl):S20-S27, 2010

9. Lindquist C, Paddick I: The Leksell Gamma Knife Perfexion and comparisons with its predecessors. Neurosurgery 61 (3 Suppl):130-141, 2007

10. Ma L, Mason E, Sneed PK, McDermott M, Polishchuk A, Larson DA, et al: Clinical realization of sector beam intensity modulation for Gamma Knife radiosurgery: a pilot treatment planning study. Int J Radiat Oncol Biol Phys 91:661-668, 2015

11. Ma L, Nichol A, Hossain S, Wang B, Petti P, Vellani R, et al: Variable dose interplay effects across radiosurgical apparatus in treating multiple brain metastases. Int J CARS 9:10791086, 2014

12. Ma L, Petti P, Wang B, Descovich M, Chuang C, Barani IJ, et al: Apparatus dependence of normal brain tissue dose in stereotactic radiosurgery for multiple brain metastases. J Neurosurg 114:1580-1584, 2011

13. Ma L, Pinnaduwage D, McDermott M, Sneed PK: Whole- procedural radiological accuracy for delivering multi-session Gamma Knife radiosurgery with a relocatable frame system. Technol Cancer Res Treat 13:403-408, 2014

14. Ma L, Sahgal A, Hwang A, Hu W, Descovich M, Chuang C, et al: A two-step optimization method for improving multiple brain lesion treatments with robotic radiosurgery. Technol Cancer Res Treat 10:331-338, 2011

15. Ma L, Sahgal A, Larson DA, Pinnaduwage D, Fogh S, Barani I, et al: Impact of millimeter-level margins on peripheral normal brain sparing for Gamma Knife radiosurgery. Int J Radiat Oncol Biol Phys 89:206-213, 2014

16. Ma L, Xia P, Verhey LJ, Boyer AL: A dosimetric comparison of fan-beam intensity modulated radiotherapy with Gamma Knife stereotactic radiosurgery for treating intermediate intracranial lesions. Int J Radiat Oncol Biol Phys 45:13251330, 1999

17. Ma Y, Li M, Yin Y, Kong L, Sun X, Lin X, et al: Hypofractionated stereotactic radiotherapy for brain metastases: a dosimetric and treatment efficiency comparison between volumetric modulated arc therapy and intensity modulated radiotherapy. Technol Cancer Res Treat 9:499507,2010

18. Otto K: Volumetric modulated arc therapy: IMRT in a single gantry arc. Med Phys 35:310-317, 2008

19. Paddick I: A simple scoring ratio to index the conformity of radiosurgical treatment plans. Technical note. J Neurosurg 93 (Suppl 3):219-222, 2000

20. Paddick I, Lippitz B: A simple dose gradient measurement tool to complement the conformity index. J Neurosurg 105 Suppl:194-201, 2006

21. Régis J, Tamura M, Guillot C, Yomo S, Muraciolle X, Nagaje M, et al: Radiosurgery with the world's first fully robotized Leksell Gamma Knife PerfeXion in clinical use: a 200-patient prospective, randomized, controlled comparison with the Gamma Knife 4C. Neurosurgery 64:346-356, 2009

22. Ruschin M, Komljenovic PT, Ansell S, Ménard C, Bootsma G, Cho YB, et al: Cone beam computed tomography image guidance system for a dedicated intracranial radiosurgery treatment unit. Int J Radiat Oncol Biol Phys 85:243-250, 2013

23. Sahgal A, Aoyama H, Kocher M, Neupane B, Collette S, Tago M, et al: Phase 3 trials of stereotactic radiosurgery with or without whole-brain radiation therapy for 1 to 4 brain metastases: individual patient data meta-analysis. Int J Radiat Oncol Biol Phys 91:710-717, 2015

24. Sahgal A, Ma L, Chang E, Shiu A, Larson DA, Laperriere $\mathrm{N}$, et al: Advances in technology for intracranial stereotactic radiosurgery. Technol Cancer Res Treat 8:271-280, 2009

25. Schell MC, Bova FJ, Larson DA, Leavitt DD, Lutz WR, Podgorsak EB, et al: AAPM Report No. 54. Stereotactic Radiosurgery. Woodbury, NY: American Institute of Physics, 1995

26. Schlesinger D, Xu Z, Taylor F, Yen CP, Sheehan J: Interfraction and intrafraction performance of the Gamma Knife Extend system for patient positioning and immobilization. J Neurosurg 117 Suppl:217-224, 2012

27. Soltys SG, Kirkpatrick JP, Laack NN, Kavanagh BD, Breneman JC, Shih HA: Is less, more? The evolving role of radiation therapy for brain metastases. Int J Radiat Oncol Biol Phys 92:963-966, 2015

28. Thomas EM, Popple RA, Prendergast BM, Clark GM, Dobelbower MC, Fiveash JB: Effects of flattening filter-free and volumetric-modulated arc therapy delivery on treatment efficiency. J Appl Clin Med Phys 14:4328, 2013

29. Thomas EM, Popple RA, Wu X, Clark GM, Markert JM, Guthrie BL, et al: Comparison of plan quality and delivery time between volumetric arc therapy (RapidArc) and Gamma Knife radiosurgery for multiple cranial metastases. Neurosurgery 75:409-418, 2014

30. Vanetti E, Nicolini G, Nord J, Peltola J, Clivio A, Fogliata A, et al: On the role of the optimization algorithm of RapidArc ${ }^{\circledR}$ 
volumetric modulated arc therapy on plan quality and efficiency. Med Phys 38:5844-5856, 2011

31. Wolff D, Stieler F, Welzel G, Lorenz F, Abo-Madyan Y, Mai $\mathrm{S}$, et al: Volumetric modulated arc therapy (VMAT) vs. serial tomotherapy, step-and-shoot IMRT and 3D-conformal RT for treatment of prostate cancer. Radiother Oncol 93:226-233, 2009

32. Yu CX, Tang G: Intensity-modulated arc therapy: principles, technologies and clinical implementation. Phys Med Biol 56:R31-R54, 2011

\section{Disclosures}

Professor Ma is a patent holder for the University of California Regents.

\section{Author Contributions}

Conception and design: Ma, McDermott, Sneed. Acquisition of data: Ma, Dong, Pérez-Andújar, Pinnaduwage, Theodosopoulos. Analysis and interpretation of data: all authors. Drafting the article: Ma, Dong, Pérez-Andújar, Pinnaduwage, Braunstein. Critically revising the article: Ma, Dong, Pérez-Andújar, Pinnaduwage, Braunstein. Reviewed submitted version of manuscript: all authors. Approved the final version of the manuscript on behalf of all authors: Ma. Administrative/technical/ material support: Ma, Theodosopoulos, McDermott, Sneed. Study supervision: Ma, McDermott, Sneed.

\section{Correspondence}

Lijun Ma, Department of Radiation Oncology, University of California, San Francisco, 505 Parnassus Ave., Rm. L-08, San Francisco, CA 94143. email: lijun.ma@ucsf.edu. 